\title{
Nanoscale
}

A) Check for updates

Cite this: Nanoscale, 2020, 12, 17064

\section{Rapid synthesis of a BiaZIF-8 composite nanomaterial as a near-infrared-II (NIR-II) photothermal agent for the low-temperature photothermal therapy of hepatocellular carcinoma}

\author{
Jinghua Li, $\dagger^{\mathrm{a}}$ Daoming Zhu, (D) $\dagger^{\mathrm{b}}$ Weijie Ma, ${ }^{a}$ Yang Yang, ${ }^{\mathrm{b}}$ Ganggang Wang, ${ }^{\mathrm{a}}$ \\ Xiaoling Wu, ${ }^{a}$ Kunlei Wang, ${ }^{a}$ Yiran Chen, ${ }^{a}$ Fubing Wang, (D) ${ }^{d}$ Wei Liu (D) ${ }^{b}, c$ and \\ Yufeng Yuan (iD *a
}

\begin{abstract}
Hepatocellular carcinoma is the fourth leading cause of cancer-related deaths globally. Advanced nanomaterials have emerged as effective approaches to liver cancer therapy such as photothermal therapy. However, limited penetration depth of photothermal agents (PTAs) activated in the NIR-I bio-window and thermoresistance due to heat shock proteins restrict the therapeutic efficacy of PTT in HCC. Herein, we prepared a BiaZIF-8 (BZ) nanomaterial by a simple one-step reduction method. Then, gambogic acid, a natural inhibitor of Hsp90, was efficiently loaded onto the BZ nanomaterial via physical mixing. The characterization of the nanomaterial and release of GA due to $\mathrm{pH}$ change or NIR-light irradiation were separately studied. Photothermal conversion efficiency was calculated, and therapeutic studies were carried out in vitro and in vivo. This nanomaterial exhibited a significantly enhanced drug release rate when the temperature was increased under acidic conditions and had good light stability under laser irradiation and a photothermal conversion efficiency of about $24.4 \%$. In addition, this novel nanomaterial achieved good therapeutic effects with less toxicity in vitro. The BZ nanomaterial loaded with GA caused tumor shrinkage as well as disappearance and effectively downregulated Hsp90 expression in tumors in vivo. Moreover, this novel nanomaterial exhibited good biocompatibility and potential for application in low-temperature PTT with excellent tumor destruction efficacy.
\end{abstract}

\begin{abstract}
Received 21st May 2020 Accepted 23rd July 2020 DOI: $10.1039 /$ d0nr03907a rsc.li/nanoscale
\end{abstract}

\section{Introduction}

Hepatocellular carcinoma (HCC) is the fourth most common cancer and ranked as one of the leading causes of cancerrelated deaths globally. ${ }^{1}$ Chronic $\mathrm{HBV}$ and $\mathrm{HCV}$ infections are the main causes of HCC and account for $80 \%$ of the HCC global cases. ${ }^{2}$ Treatment of the disease depends on the stage of the HCC. Due to the limited aggressiveness of early-stage HCC, clinical guidelines recommend surgical therapies as the first line treatment. ${ }^{3,4}$ However, for most patients in the middle and advanced stages, no effective treatment is available as they are often unfit for surgery. These patients mainly

\footnotetext{
${ }^{a}$ Department of Hepatobiliary and Pancreatic Surgery, Zhongnan Hospital of Wuhan University, Wuhan 430071, China. E-mail: yuanyf1971@whu.edu.cn

${ }^{b}$ Key Laboratory of Artificial Micro- and Nano-Structures of Ministry of Education

School of Physics and Technology, Wuhan University, Wuhan 430071, China

${ }^{c}$ Wuhan University Shenzhen Institution, Shenzhen 518057, China

${ }^{d}$ Department of Laboratory Medicine, Zhongnan Hospital of Wuhan University,

Wuhan 430071, China

$\dagger$ These authors contributed equally.
}

receive palliative treatments such as transhepatic arterial chemoembolization (TACE), radiofrequency ablation (RFA), and percutaneous ethanol injection (PEI). However, these treatments have limited efficacy and can exacerbate underlying liver disease partly due to HCC. In addition, portal vein tumor thrombosis (PVTT) has been reported in about $35-50 \%$ of patients. This is characterized by a strong negative prognostic factor owing to high malignancy in bloodstream, resulting in limited treatment options and high recurrence risk of HCC. ${ }^{5,6}$ Therefore, an effective and harmless treatment of hepatocellular carcinoma is urgently required.

As promising emergent strategies, photothermal therapy (PTT) and photodynamic therapy are currently under intensive research in preclinical and clinical cancer treatments where high temperature is generated to kill tumor cells. ${ }^{7-9}$ The damaged tumor cells stimulate the immune system of the patient; this leads to the proliferation and differentiation of immune cells as well as induction of tumor cell necrosis and apoptosis. ${ }^{10,11}$ These therapies play a role in killing tumors and have good application prospects in the treatment of cancer. Recently, in PTT, photothermal agents (PTAs) have 
been extensively explored under near-infrared (NIR) laser irradiation for tumor hyperthermia ablation. ${ }^{12-14}$ Most importantly, the wavelength of the NIR light is located in the "biological window", and NIR light is negligibly absorbed by the blood and soft tissues; this causes its deep penetration in tissues. ${ }^{13,15,16}$ In addition, most of the PTAs are active in the first NIR (NIR-I) region, and the restricted penetration depth of the laser affects the therapeutic efficacy of these PTAs. ${ }^{17}$ Alternatively, using a laser with two near-infrared zones (NIR-I (750-1000 nm) and NIR-II (1000-1350 nm)), especially within 1000-1100 nm, deeper penetration and higher maximum allowable exposure (MPE) of PTAs can be achieved as compared to the case of the laser with only the NIR-I bio-window (750-1000 nm). This significantly improves the light-to-heat conversion efficiency of PTAs. ${ }^{18,19}$ In PTT, to achieve thorough tumor ablation, rigorous photothermal heating to over $50{ }^{\circ} \mathrm{C}$ is required to induce complete cell necrosis. ${ }^{20}$ Furthermore, high-temperature tumor ablation under strong laser irradiation may cause damage to normal organs near the tumor due to laser-induced nonspecific heating..$^{21,22}$ Moreover, cell damage (e.g. apoptosis) due to heating at lower temperatures $\left(\right.$ e.g. $\left.45^{\circ} \mathrm{C}\right)$ can be repaired with the aid of heat shock proteins (Hsp) ${ }^{23}$ However, the delivery of sufficient heat to the internal part of large tumors or deeply located tumors might not be possible, leading to the possible survival of tumor cells after the PTT and their subsequent spread to other organs. ${ }^{24}$ Therefore, the development of NIR-II photothermal therapy strategies to effectively destroy tumors under low-temperature heating is important for the future clinical transformation of hepatocellular carcinoma treatment methods.

The currently available PTAs, such as $\mathrm{Au}^{25-27} \mathrm{Ag},{ }^{28-30}$ and $\mathrm{Pd}^{31-33}$ nanoparticles, have been extensively investigated. However, these metals are rare and expensive, limiting their clinical large-scale application. Specifically, bismuth(Bi), a typical semi-metal element, has aroused great interest from scientific researchers. ${ }^{34-36}$ Bismuth is a well-known "green metal" that is nontoxic and inexpensive. ${ }^{37}$ The recent discovery of plasmonic properties that originate from the semimetal-to-semiconductor transition (also called nanoconfinement effects) of Bi at nanoscale has extended the possible applications of Bi-based nanomaterials (traditionally used as thermoelectric materials, catalysts, and sensors) as cancer therapeutic agents in nanomedicine. ${ }^{38,39}$ Studies have demonstrated that Bi can also be used as a radiosensitizer and contrast agent in computed tomography (CT)..$^{40,41}$ Due to these potential biomedical applications, bismuth nanoparticles can be fabricated as photothermal agents for the effective treatment of HCC.

Herein, we designed a novel strategy to synthesize a nanomaterial containing bismuth nanodots embedded in ZIF-8 nanoparticles (BZ). ZIF-8 was used as an excellent reaction container that quickly encapsulated the bismuth nanodots and acted as a small-molecule drug carrier with outstanding drug loading efficiency. ${ }^{42,43}$ Furthermore, BZ was loaded with gambogic acid (GBZ), a natural inhibitor of heat-shock protein 90 (Hsp90), for NIR-II low-temperature photothermal therapy. ${ }^{44}$ Since Hsp90 is a key protein that induces thermoresistance in cells under hyperthermia, ${ }^{45}$ the inhibition of Hsp90 by GA delivered using BZ effectively induced the apoptosis of cancer cells under low-temperature heating $\left(\right.$ e.g. $\left.43^{\circ} \mathrm{C}\right)$.

To the best of our knowledge, to date, no study has been reported on the photothermal properties of Bi nanoparticles and GA in hepatocellular carcinoma treatment and the further development of Bi nanoparticles and GA for biomedical applications. The composite nanomaterials prepared herein successfully expanded the application of $\mathrm{Bi}$ in the treatment of hepatocellular carcinoma and provided more opportunities for the successful clinical application of PTT.

\section{Results and discussion}

\section{1. Characterization of GBZ}

At first, we prepared ZIF-8 nanoparticles coated with bismuth nanodots via a simple reduction method and then loaded gambogic acid onto them through physical mixing. The entire synthesis step did not require complicated conditions, and the products were synthesized in large quantities. Subsequently, we characterized the products using electron microscopy. Our results revealed that the particle size of GBZ was approximately $100 \mathrm{~nm}$. The small size of GBZ was due to the short reaction time and fast stirring speed. In addition, there were apparent black nanodots in GBZ; this indicated that the bismuth nanodots were successfully fixed on ZIF-8 (Fig. 1A). Scanning electron microscopy results also indicated that the nanoparticles were synthesized in large quantities (Fig. 1C). To demonstrate that the bismuth nanoparticles were successfully immobilized onto ZIF-8, we performed element analysis (Fig. 1B) and energy spectrum analysis (Fig. 1D). Our analysis results revealed that bismuth was present in ZIF-8. The loading content of gambogic acid (LC) was calculated to be $31.4 \%$. XRD (Fig. 1E) results showed that the synthesized GBZ atlas match the ZIF-8 and Bi standard atlas. The hydrodynamic diameter of the GBZ nanoparticles was approximately $100 \mathrm{~nm}$ (Fig. 1F). Overall, GBZ was successfully synthesized in large quantities and was used in subsequent experiments.

\subsection{In vitro photothermal properties of the GBZ nanoparticles}

We verified the photothermal conversion efficiency of GBZ in vitro. The UV-vis-NIR absorption spectrum (Fig. 2A) indicated that gambogic acid has a relatively strong absorption peak near $240 \mathrm{~nm}$, whereas GBZ has a strong absorption peak at $240 \mathrm{~nm}$, which indicated that the drug was successfully loaded onto the nanoparticles. Moreover, we found that GBZ has a relatively optimal absorption in the near-infrared second region. The ZIF-8, BZ, and GBZ groups were irradiated using a $1064 \mathrm{~nm}$ laser for $1 \mathrm{~min}, 2 \mathrm{~min}$, and $3 \mathrm{~min}$, respectively. Consequently, the infrared thermal phase diagram (Fig. 2B) revealed that the BZ and GBZ groups attained a higher temperature of approximately $40{ }^{\circ} \mathrm{C}$ in a short period of time. The infrared camera detected the temperature changes of the GBZ solution at different concentrations $\left(50,100\right.$, and $\left.200 \mu \mathrm{g} \mathrm{mL} \mathrm{m}^{-1}\right)$ 
A

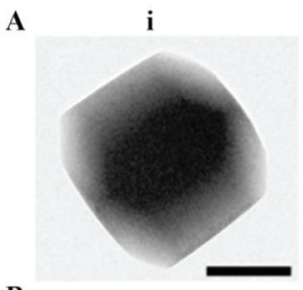

B

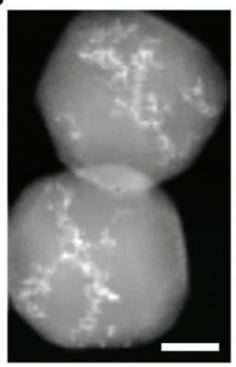

ii

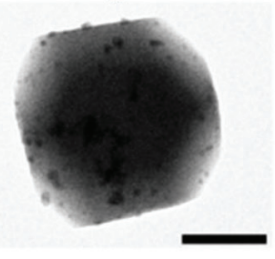

Zn

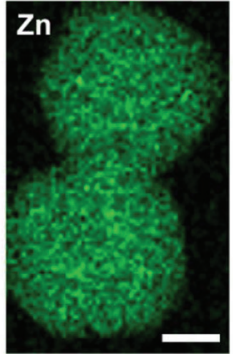

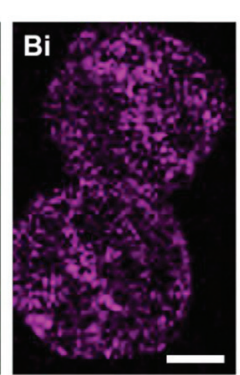

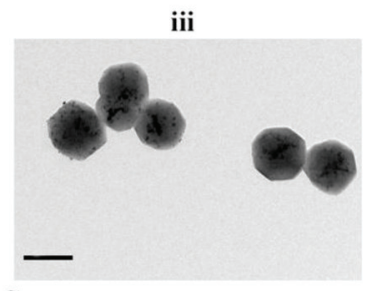

C

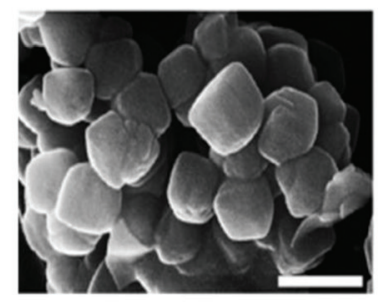

D
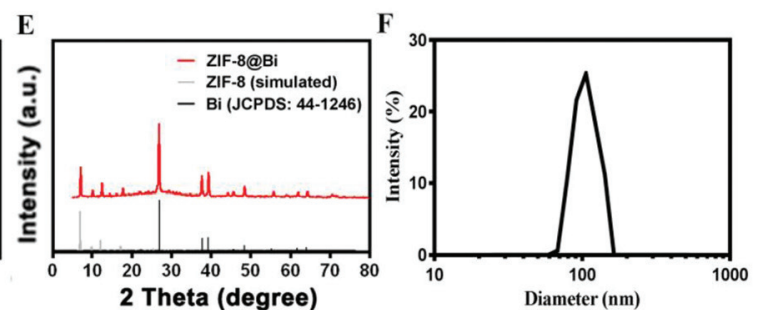

Fig. 1 (A) i. High-magnification transmission electron microscopy image of ZIF-8 nanoparticles, scale bar: $30 \mathrm{~nm}$; ii. high-magnification transmission electron microscopy image of GBZ nanoparticles, scale bar: $30 \mathrm{~nm}$; iii. low-magnification transmission electron microscopy image of GBZ nanoparticles, scale bar: $100 \mathrm{~nm}$; (B) elemental mapping images of $Z n$ and Bi, scale bar: $1 \mu \mathrm{m}$; (C) scanning electron microscopy image of the GBZ nanoparticles, scale bar: $150 \mathrm{~nm}$; (D) energy spectrum analysis of $B Z$; $(E)$ powder XRD pattern of BZ; and (F) hydrodynamic diameter of the GBZ nanoparticles measured via DLS.
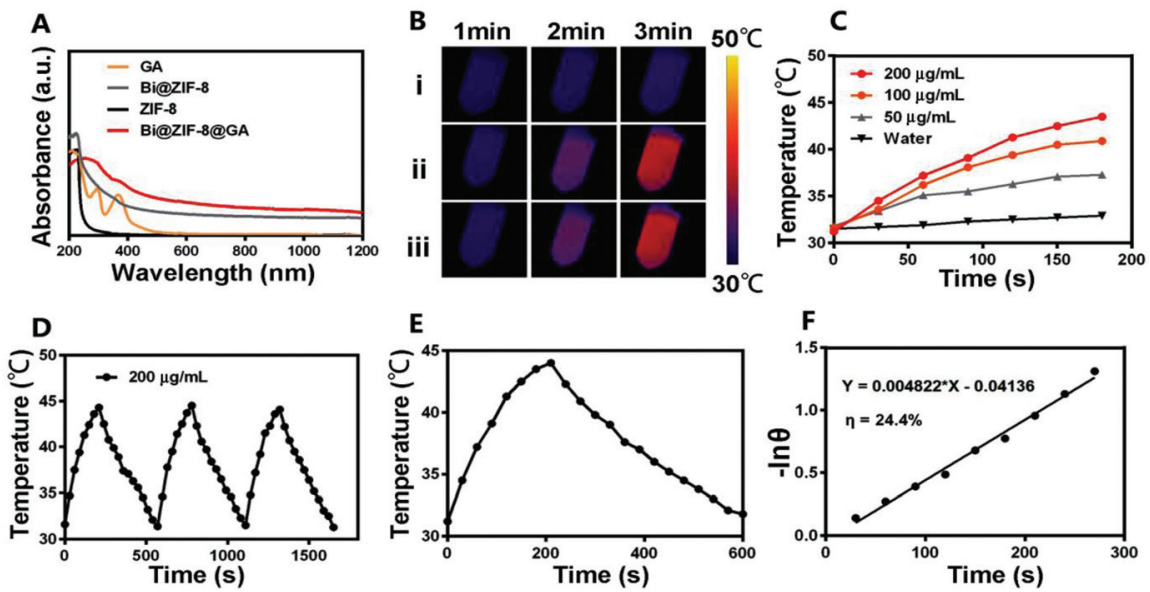

Fig. 2 (A) Absorbance spectra of GA, ZIF-8, BZ, and GBZ. (B) The infrared thermal images of (i) ZIF-8, (ii) BZ, and (iii) GBZ nanoparticles (containing $100 \mu \mathrm{g} \mathrm{mL} \mathrm{L}^{-1} \mathrm{BZ}$ ) irradiated for $1-3 \mathrm{~min}\left(1064 \mathrm{~nm}, 1 \mathrm{~W} \mathrm{~cm}^{-2}\right)$; (C) temperature rise curve of the $\mathrm{GBZ}$ solution at different concentrations (50, 100, and $200 \mu \mathrm{g} \mathrm{mL}^{-1}$ ) irradiated by a $1064 \mathrm{~nm}$ laser at $1 \mathrm{~W} \mathrm{~cm}{ }^{-2}$ for $5 \mathrm{~min}$; (D) the photothermal response of a $200 \mu \mathrm{g} \mathrm{mL}^{-1} \mathrm{GBZ}_{\mathrm{Banoparticle}}$ aqueous solution analyzed using a NIR laser $\left(1064 \mathrm{~nm}, 1 \mathrm{~W} \mathrm{~cm}^{-2}\right)$; then, the laser was turned off, and the process was repeated three times; (E) the photothermal response of an aqueous solution containing $200 \mu \mathrm{g} \mathrm{mL} \mathrm{L}^{-1} \mathrm{GBZ}$ nanoparticles measured using a NIR laser $(1064 \mathrm{~nm}, 1 \mathrm{~W} \mathrm{~cm}$ ), and then, the laser was shut off; and (F) linear time data versus $-\ln \theta$ obtained from the cooling period shown in (E).

and water under $1064 \mathrm{~nm}$ laser irradiation (Fig. 2C). With an increase in the nanoparticle concentration and irradiation time, the trend of the curve increased significantly. In the case

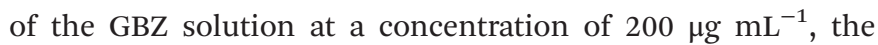
temperature increased to approximately $42{ }^{\circ} \mathrm{C}$ after $150 \mathrm{~s}$, implying that the GBZ solution had profound light stability 
under laser irradiation. To confirm that the GBZ solution had profound photothermal cycling stability and high photothermal conversion efficiency (Fig. 2D), the $200 \mu \mathrm{g} \mathrm{mL}{ }^{-1} \mathrm{GBZ}$ solution was subjected to heating and cooling cycles, and it remained stable after three cycles of heating and cooling. Fig. 2E shows the recorded temperature change of the GBZ solution under continuous irradiation of a $1064 \mathrm{~nm}$ laser. Based on the experimental data and the method reported in previous studies (Fig. 2E), the photothermal conversion efficiency of GBZ was approximately $24.4 \%$ (Fig. 2F), which was lower than that of the recently reported bismuth-based nanomaterials $(30 \%))^{34}$ but was higher than those of some photothermal agents such as Au nanodots $(21 \%)^{46}$ and $\mathrm{Cu}_{2-x} \mathrm{Se}(22 \%) .{ }^{47}$ Overall, these data indicated that GBZ is a profound nanomaterial for photothermal treatment.

\subsection{Verification of the GBZ drug release characteristics}

Next, we verified the GBZ drug release characteristics. Our findings revealed that under no-light conditions, the ZIF-8 nanomaterial had a profound $\mathrm{pH}$ response, and the GBZ drug release rate was markedly faster than that under neutral and acidic conditions (Fig. 3A). After light exposure, faster drug release rates were observed because of the increase in the temperature of the nanomaterial. These results demonstrated that the BZ nanomaterials are suitable for drug loading and release, which are crucial for PTT.

\subsection{Anti-tumor effect of GBZ in vitro}

Based on the profound photothermal conversion efficiency of GBZ, we further verified its ability in apoptotic cancer cells in vitro. The CCK-8 experiment (Fig. 3B) validated the toxicity of Huh7 cells in each group under light conditions. With an increase in material concentration under light, the rate of cell survival further decreased. GBZ hindered cell growth at approximately $85 \%$ when compared with the case of the PBS group. The inhibitory effect of BZ on cell growth under light was about $60 \%$. Moreover, GA had some anti-tumor ability. In the absence of light irradiation, whether the nanomaterials have an apoptotic effect on normal cells or tumor cells is unclear. We further investigated the toxicity of BZ in normal liver cells, immune cells, and tumor cells. Consequently, all types of cells had higher survival rates (over 90\%) at each concentration of BZ (Fig. 3C). Therefore, the BZ nanomaterials are less toxic and safe for use in in vivo experiments.

To further study the efficacy of GBZ in in vitro treatment, we used $35 \mathrm{~mm}$ cell culture dishes to culture Huh-7 cells to $1 \times$ $10^{5}$ cells per $1 \mathrm{~mL}$ followed by the addition of PBS $(50 \mu \mathrm{g}$ $\mathrm{mL}^{-1}$ ) and BZ solution (70 $\left.\mu \mathrm{g} \mathrm{mL}^{-1}\right)$, respectively. A GA solution $\left(30 \mu \mathrm{g} \mathrm{mL}{ }^{-1}\right)$ and GBZ solution (containing $70 \mu \mathrm{g} \mathrm{mL}{ }^{-1} \mathrm{BZ}$ and $30 \mu \mathrm{g} \mathrm{mL}{ }^{-1} \mathrm{GA}$ ) were co-cultured with Huh-7 cells for an additional $12 \mathrm{~h}$ and then irradiated with a $1064 \mathrm{~nm}$ laser for $3 \mathrm{~min}$. Then, we stained the cells using fluorescein diacetate (FDA) and propidium iodide (PI) for $5 \mathrm{~min}$. Finally, the fluorescence images were obtained using a fluorescence microscope (Olympus, Japan), as shown in Fig. 3D. Our findings indicated that almost all the Huh-7 cells in the PBS group emitted green fluorescence, implying that an insignificant number of Huh-7 cells were killed. Part of the Huh-7 cells in the BZ group and GA group emitted red fluorescence; in the GBZ group, almost all the Huh-7 cells emitted red fluorescence, implying that all the Huh-7 cells were dead. Overall, these results showed that the combination of BZ with GA has

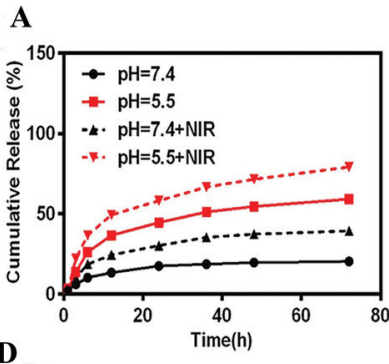

B
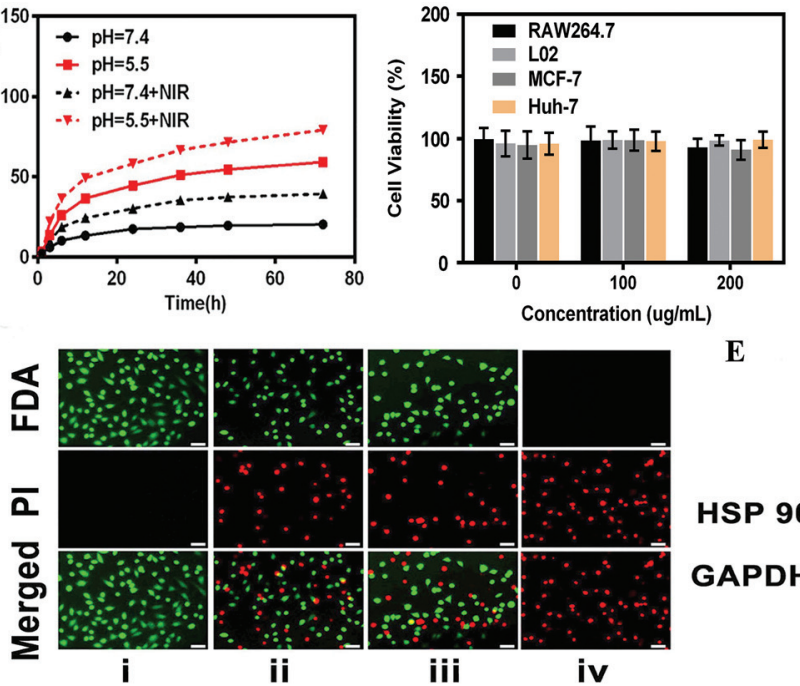

$\mathbf{E}$
C
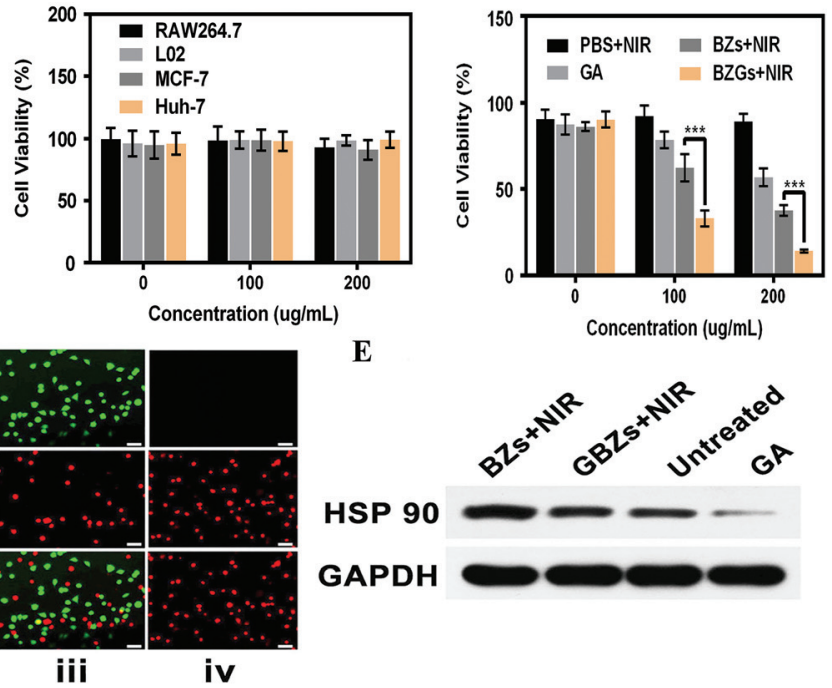

Fig. 3 (A) NIR-triggered $\left(1 \mathrm{~W} \mathrm{~cm}^{-2}\right.$ ) release of GA from GBZ at a pH of 7.4 and 5.5; (B) in vitro cytotoxicity of different nanomaterials against Huh-7 cells in the presence of light irradiation (1064 nm, $\left.1 \mathrm{~W} \mathrm{~cm}^{-2}, 5 \mathrm{~min}\right), * \star * p<0.01$; (C) in vitro cytotoxicity of BZ against RAW264.7, L02, MCF-7, and Huh-7 cell lines in the absence of light irradiation; (D) fluorescence images of the Huh-7 cells co-stained with FDA (live cells, green) and PI (dead cells, red) upon the addition of different nanomaterials under irradiation (1064 nm, $1 \mathrm{~W} \mathrm{~cm}^{-2}, 3 \mathrm{~min}$ ). (i) PBS, (ii) GA, (iii) BZ, and (iv) GBZ; scale bar: $50 \mu \mathrm{m}$; (E) western blot images: Hsp90 expression levels with GADPH as an internal reference for the cell lysates of Huh-7 cells after various treatments. 
optimal therapeutic effects in vitro. We examined the Hsp90 expression in Huh-7 cells under different experimental treatments via western blot assays (Fig. 3E) using glyceraldehyde 3-phosphate dehydrogenase (GAPDH) as a loading control. The results indicated that the Huh-7 cells under hyperthermia treatment had higher levels of $\mathrm{Hsp} 90$ at $43{ }^{\circ} \mathrm{C}$ when compared with the case of the untreated control cells. The treatment of the cells with GA-loaded nanoparticles effectively inhibited this elevated expression of Hsp90.

\subsection{In vivo photothermal cancer therapy}

Currently, photothermal therapy is the most extensively researched therapy, and in vivo photothermal efficacy is the most direct validation approach used for assessing suitable nanoparticles for photothermal therapy. ${ }^{48-50}$ Therefore, we validated the photothermal efficacy of GBZ nanomaterials in vivo. We used an infrared thermal imaging camera to monitor the photothermal effect of these nanomaterials in the body by concurrently plotting the temperature changes at the site of the tumor. Consequently, we found that the temperature at the site of the tumor is positively associated with the irradiation period after the injection of the $\mathrm{Bi}$ nanodots. ${ }^{51}$ However, we did not observe any evident temperature changes in the case of the control mice (Fig. 4A). In Fig. 4B, the temperature in the control group is almost constant. The temperature in the tumor microenvironment of the BZ and GBZ groups increased by approximately $10^{\circ} \mathrm{C}$. This indicated that the nanoparticles have an optimal photothermal conversion efficiency and can be heated to a temperature exceeding the tolerance temperature of the cells $\left(42^{\circ} \mathrm{C}\right)$. Our findings show that the Bi nanodots can potentially be utilized in the realtime monitoring of thermal dynamics in PTT.

We measured the tumor volume and body weight of each group in this study every two days to assess the anti-tumor effect of BZ following the photothermal treatment of tumor sites in mice. Our results revealed rapid tumor growth in the PBS group, and the tumor expanded from about $200 \mathrm{~mm}^{3}$ to $780 \mathrm{~mm}^{3}$ (Fig. 4C and D). Tumor growth in the BZ group was substantially slower than that in the PBS group. Moreover, the tumors in the GBZ group were gradually shrinking or even disappearing. There was no evident variation in the body weight in all the groups under different treatments with prolonged irradiation time, indicating no noticeable systemic toxicity of the proposed photothermal molecule. The mean weights of the excised tumors are shown in Fig. 4E. Notably, the weights and volumes of the tumors exhibited a similar trend. We randomly selected one mouse per group for PET-CT. Consequently, the metabolism at the tumor site was strongest in the case of the PBS group and weakest in the case of the GBZ group (Fig. 5A). Interestingly, the tumor metabolism of the laser-irradiated GBZ group was higher than that of the group without laser irradiation. This could be attributed to individual differences in mice. These results confirmed that GBZ has significant potential as an ideal PTT agent for the in vivo photothermal ablation of tumors.

Furthermore, we harvested the tumor tissues in all the groups for histological examinations via H\&E, Ki-67, and TUNEL staining. Moreover, we harvested the tumors for immunefluorescence staining to assay the expression levels of Hsp90. The H\&E staining results showed that the tumor tissue of the mice treated with both the GBZ injection and laser

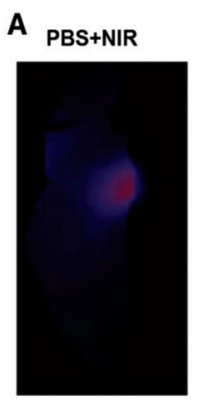

C
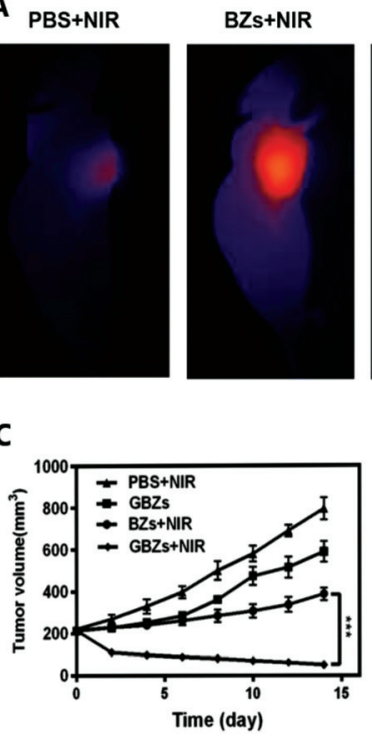

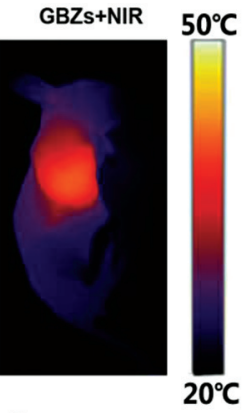

D

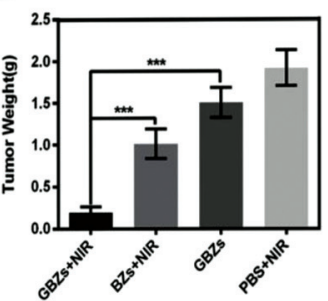

B
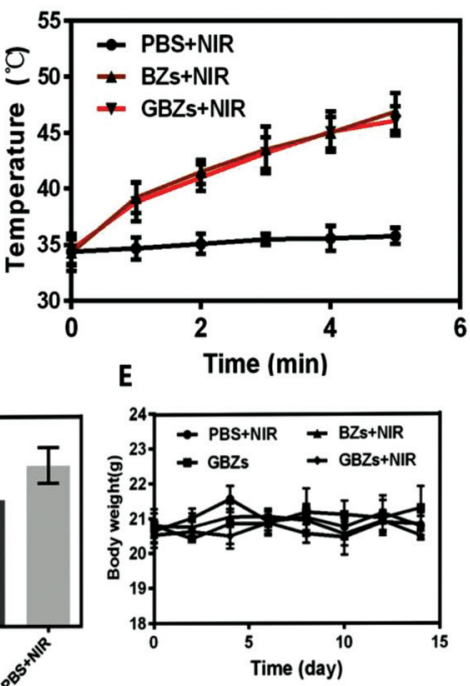

Fig. 4 (A) Infrared thermal images and (B) temperature curves of the tumor-bearing mice intravenously injected with PBS (control), BZ, and GBZ, and then irradiated with an $808 \mathrm{~nm}$ NIR laser $\left(808 \mathrm{~nm}, 1 \mathrm{~W} \mathrm{~cm}{ }^{-2}\right)$ for $5 \mathrm{~min}$; (C) tumor volume of the mice in different groups after various treatments; (D) tumor weight of the mice in different groups after various treatments, ${ }^{\star *} p<0.01$; and (E) body weight of the mice in different groups after various treatments. 
A

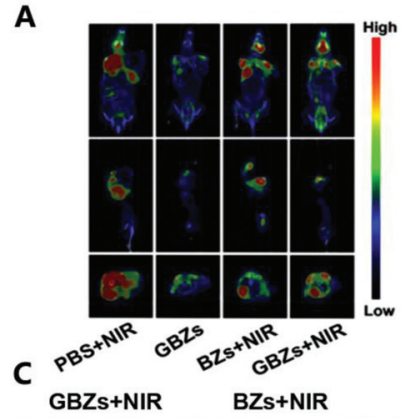

B

또
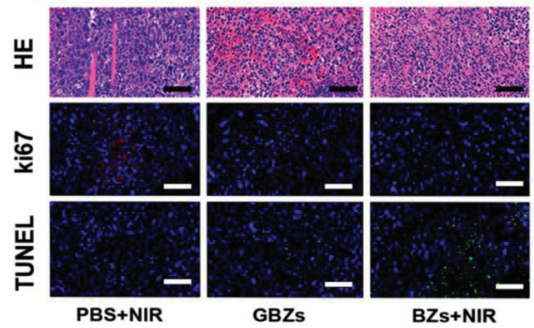

GBZs

D

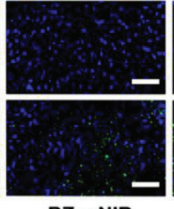

BZs+NIR

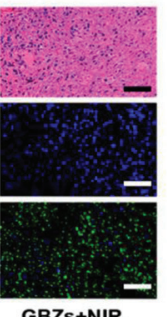

GBZs+NIR
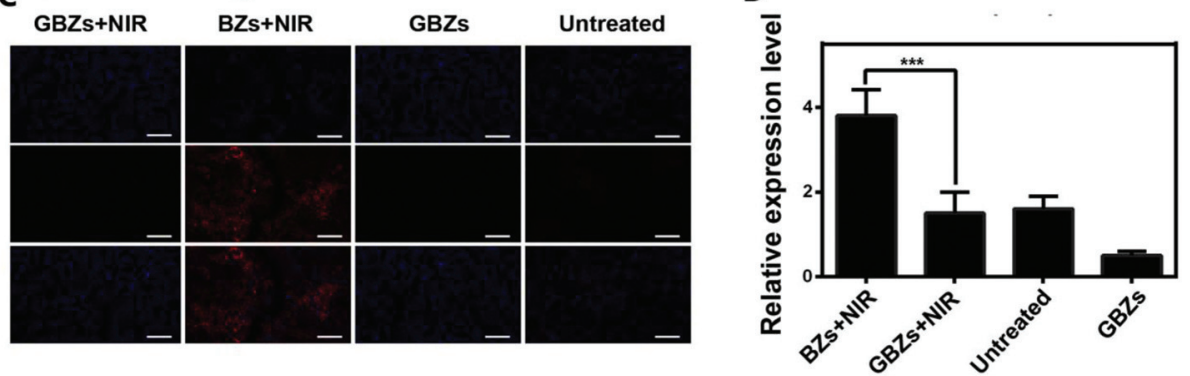

Fig. 5 (A) In vivo PET-CT and the responding degree of metabolism of mice after intravenous injection of different materials; (B) H\&E, Ki-67, and TUNEL staining images of the tumors dissected from different groups, scale bar: $200 \mu \mathrm{m}$; (C) HSP immunofluorescence staining image, scale bar: $200 \mu \mathrm{m}$; and (D) quantified immunofluorescence Hsp90 expression levels, ${ }^{* *} p<0.01$.

irradiation was remarkably damaged (Fig. 5B) followed by the tumor tissue of the mice belonging to the BZ-laser group; however, the residual tumor tissues in the rest of the groups had no evident damage. The TUNEL staining results revealed a similar trend as the H\&E staining results, and the tumor cells of the GBZ-laser group showed significant apoptosis. The Ki-67 staining results indicated the proliferation of tumor cells as expected; however, the proliferation of tumor cells in the GBZlaser group was markedly inhibited. We separated the tumors dissected from the various treatment groups for immunofluorescence staining to determine the expression level of Hsp90 in the tumors. The expression of Hsp90 was up-modulated in the tumor after the PTT treatment; this demonstrates that the PTTinduced heating stress enhances the Hsp90 expression in cancer cells in vivo (Fig. 5C). Compared with the expression level of Hsp90 in the GBZ group, the expression level of Hsp90 in the BZ group was almost twice higher (Fig. 5D), which was suppressed by treatment with the GA-loaded nanoparticles. Our results collectively indicated that the GBZ treatment downmodulates the expression of Hsp90 within tumors, resulting in a remarkably reduced thermoresistance of cancer cells during the PTT. This significantly promotes the apoptosis of cancer cells and allows low-temperature PTT with profound tumor destruction efficacy.

\section{Experimental}

\subsection{Materials}

Phosphate buffer solution (PBS) and Dulbecco's modified Eagle's medium (DMEM, Hyclone, high glucose) were obtained from Thermo-Fisher (Waltham, MA, USA). 1, 2-Distearoyl-sn- glycero-3-phosphoethanolamine- $\mathrm{N}$-[Cy5 (poly-ethylene glycol)2000] (ammonium salt) (DSPE-PEG-Cy5) was acquired from Avanti Polar Lipids (USA). All aqueous solutions were prepared using deionized (DI) water purified using an experimental water purification system (Direct-Q3, Millipore, USA). Analytical-grade bismuth nitrate pentahydrate (99\%) (Bi $\left.\left(\mathrm{NO}_{3}\right)_{3} \cdot 5 \mathrm{H}_{2} \mathrm{O}\right)$, gambogic acid (GA), zinc nitrate hexahydrate $\left(\mathrm{Zn}\left(\mathrm{NO}_{3}\right)_{2} \cdot 6 \mathrm{H}_{2} \mathrm{O}\right)$, sodium borohydride $\left(\mathrm{NaBH}_{4}\right)$, and 2-methylimidazole (MeIm) were purchased from Aladdin-Reagent (Shanghai, China). All the chemicals were of analytical grade and were used without further purification.

\subsection{Cell line processing}

The human hepatocellular carcinoma cell lines Huh-7, L02, and MCF-7 were cultured in Dulbecco's modified Eagle's medium (DMEM) supplemented with $10 \%$ bovine serum (FBS) at $37{ }^{\circ} \mathrm{C}$ under a humidified $5 \% \mathrm{CO}_{2}$ atmosphere. The mouse leukocyte cell line RAW264.7 was cultured in a medium supplemented with $10 \%$ FBS at $37{ }^{\circ} \mathrm{C}$ and $5 \% \mathrm{CO}_{2}$.

\subsection{Preparation of Bi nanodot-encapsulated ZIF-8 nanoparticles (BZ)}

Typically, a mixture of $\mathrm{Zn}\left(\mathrm{NO}_{3}\right)_{2} \cdot 6 \mathrm{H}_{2} \mathrm{O}(100 \mathrm{mg}), \quad \mathrm{Bi}$ $\left(\mathrm{NO}_{3}\right)_{3} \cdot 5 \mathrm{H}_{2} \mathrm{O}(70 \mathrm{mg})$, and 2-methylimidazole $(1.94 \mathrm{~g})$ was dissolved in $10 \mathrm{~mL}$ of deionized (DI) water. The resulting mixture was stirred for $5 \mathrm{~min}$. Thereafter, $50 \mathrm{mg} \mathrm{NaBH}{ }_{4}$ in $1 \mathrm{~mL}$ DI water was quickly added to the abovementioned mixture followed by stirring for $1 \mathrm{~min}$. After the reaction, the products were obtained by centrifugation, washed three times with deionized water, and then vacuum-dried. The content of bismuth was quantified via ICP-AES. 


\subsection{Preparation of GA-loaded BZ (GBZ) nanoparticles}

The GBZ nanoparticles were prepared as follows: $1 \mathrm{~mL}$ GA solution ( $1 \mathrm{mg} \mathrm{mL}^{-1}$ dispersed in DMSO) was slowly added to $1 \mathrm{~mL}$ DMSO containing $1 \mathrm{mg}$ BZ followed by stirring at room temperature for $30 \mathrm{~min}$. The resulting solution was then centrifuged at $8000 \mathrm{rpm}$ for $10 \mathrm{~min}$ followed by washing with distilled water. The loading content (LC) was calculated using the following formula: LC = (weight of feeding drug - weight of redundant drug/weight of drug-loaded nanoparticles) $\times$ $100 \% .^{52}$

\subsection{Characterization}

The crystal structure and phase purity of the product were investigated by powder X-ray diffraction (XRD) using an X-ray diffractometer (D8 Advance, AXS Instruments, Germany). Transmission electron microscopy (TEM) images were obtained by Philips Tecnai-12 (the Netherlands) operated at $120 \mathrm{kV}$. The composition of the samples was studied using a field-emission scanning electron microscope (FE-SEM, S-4800, Hitachi) equipped with an energy-dispersive X-ray (EDX) spectrometer. UV-vis-NIR spectra were acquired using a UV-3600 spectrophotometer. Hydrodynamic diameters of the nanoparticles suspended in $1 \times$ PBS were measured using dynamic light scattering (DLS) (Nano-Zen 3600, Malvern Instruments, UK).

\subsection{Photothermal conversion efficiency}

A 1064 nm NIR laser (Changchun New Industries Tech. Co., Ltd, China) was used to stimulate BZ at different concentrations $\left(0,50,20,100\right.$, and $\left.200 \mu \mathrm{g} \mathrm{mL}{ }^{-1}\right)$ in the PBS solution. Photothermal images of the BZ nanoparticle-based suspensions were obtained every $30 \mathrm{~s}$ during laser irradiation using an infrared thermal imaging system. The NIR laser source was equipped with a $7 \mathrm{~mm}^{2}$ round spot laser module with adjustable power. The photothermal conversion efficiency was calculated using the following equation: ${ }^{41}$

$$
\eta=\frac{h S\left(T_{\max }-T_{\text {surr }}\right)-Q_{0}}{I\left(-10^{-A \lambda}\right)}
$$

where $h$ is the heat transfer coefficient, $S$ is the surface of the container, and $T_{\max }$ and $T_{\text {surr }}$ are the equilibrium temperature and ambient temperature, respectively. $Q_{0}$ is the heat associated with the light absorbance of the solvent, $A \lambda$ is the absorbance of the BZ nanoparticles at $1064 \mathrm{~nm}$, and $I$ is the laser power density. According to this equation, the $\eta$ value of the BZ nanoparticles was determined to be about $24.4 \%$.

\subsection{Drug release}

The release of GA due to either $\mathrm{pH}$ change or NIR-light irradiation was separately studied. To investigate the timedependent cumulative release profiles of GA-loaded GBZ at various $\mathrm{pH}$ values, $5 \mathrm{mg}$ of NPs was dispersed in $20 \mathrm{~mL}$ of buffer solution ( $\mathrm{pH} 7.4$ and 5.5, respectively) at $37{ }^{\circ} \mathrm{C}$. The resulting mixture was continuously stirred. At each time point, $1 \mathrm{~mL}$ of release medium was sampled, and the concentration of GA released into the solution was determined via UV-vis spectrophotometry. Next, the sample was returned to the original release system. Subsequently, the release profiles of DOX under $1064 \mathrm{~nm}$ laser irradiation at various $\mathrm{pH}$ values were also acquired, and the GA release was measured by UV-vis spectrophotometry as described above.

\subsection{In vitro photothermal ability of GBZ nanoparticles}

3.8.1 Live-dead cell staining. The Huh-7 cells were seeded into 6-well plates. After incubation for $24 \mathrm{~h}, \mathrm{PBS}, \mathrm{BZ}, \mathrm{GA}$, and GBZ were separately added followed by incubation for another $24 \mathrm{~h}$. Thereafter, the cells were exposed to laser irradiation ( $808 \mathrm{~nm}, 1 \mathrm{~W} \mathrm{~cm}^{-2}$ ) for $5 \mathrm{~min}$ and co-stained with $1 \mathrm{mg} \mathrm{mL}^{-1}$ propidium iodide (PI) and $5 \mathrm{mg} \mathrm{mL}^{-1}$ fluorescein diacetate (FDA) for 15 min before being washed several times with PBS. The fluorescence microscopy images were obtained using an Olympus IX81 microscope.

3.8.2 CCK-8 and western blot assays. The photothermal ablation cytotoxicity of each nanoparticle group was detected by the Cell Counting kit-8 (CCK-8, Dojindo, Japan) assay. A total of $8 \times 10^{3}$ Huh-7 cells per well were seeded into 96-well plates and cultured. After $24 \mathrm{~h}, \mathrm{PBS}, \mathrm{BZ}, \mathrm{GA}$, and GBZ were separately added and co-cultured with the Huh-7 cells for $0 \mathrm{~h}$, $24 \mathrm{~h}$, and $48 \mathrm{~h}$. Thereafter, the cells were exposed to laser irradiation ( $808 \mathrm{~nm}, 1 \mathrm{~W} \mathrm{~cm}{ }^{-2}$ ) for $5 \mathrm{~min}$ at different times. The CCK-8 solution was added as per the guidelines followed by incubation for another $2 \mathrm{~h}$. The cytotoxicity was calculated by dividing the optical density (OD) values of the treated groups $(T)$ by the OD values of the control group $(C)(T / C \times$ $100, \%)$. Proteins were extracted from the cells to detect the expression level of heat shock protein 90 (Hsp90).

3.8.3 Biocompatibility of the GBZ nanoparticles. We estimated the biocompatibility of the GBZ nanoparticles via the abovementioned CCK-8 assay. The L02, MCF-7, RAW 264.7, and Huh 7 cell lines were seeded into 96-well plates and cultured. After 24 h, GBZ was separately added and co-cultured with these cell lines for $12 \mathrm{~h}, 24 \mathrm{~h}$, and $48 \mathrm{~h}$. The cytotoxicity was calculated by dividing the optical density (OD) values of the treated groups $(T)$ by the OD values of the control group (C) $(T / C \times 100, \%)$.

\subsection{In vivo photothermal therapy}

Male BALB/c nude mice were obtained from the Chinese Academy of Medical Science (Beijing, China). The study was performed in strict accordance with the National Regulations for the Administration of Affairs Concerning Experimental Animals and was approved by the Center for Animal Experiments/Animal Biosafety Level 3 Laboratory of Wuhan University. A total of $5 \times 10^{6}$ Huh-7 cells were subcutaneously injected into the flanks of 4 -week old male BALB/c-nu mice. Treatments started when the tumor size reached $\sim 200 \mathrm{~mm}^{3}$. The mice ${ }^{20}$ were randomly divided into 4 groups: one group was intravenously injected with $200 \mu \mathrm{L}$ PBS, another group was intravenously injected with $\mathrm{BZ}$, and the remaining two groups were intravenously injected with GBZ. After $24 \mathrm{~h}$, the PBS, BZ, and GBZ group mice bearing tumors were anesthetized, and 
the entire tumors were exposed to laser irradiation ( $808 \mathrm{~nm}, 1$ $\mathrm{W} \mathrm{cm}^{-2}$ ) for $5 \mathrm{~min}$. The other GBZ group was not exposed to the laser irradiation. During laser irradiation, an infrared thermal imaging camera was used to monitor the temperature changes at the tumor sites. The changes in the tumor volume and body weight post-irradiation were measured daily. The tumor volume $V\left(\mathrm{~mm}^{3}\right)$ was calculated according to the formula: Volume $=($ tumor length $) \times(\text { tumor width })^{2} / 2$. After 14 days of injection, one mouse was selected from each group for PET examination, and other mice were sacrificed. The tumors were then extracted for H\&E, Ki-67, and terminal deoxynucleotidyl transferase dUTP nick end labeling (TUNEL) staining. Meanwhile, tumors were also extracted for immunofluorescence staining to detect the expression level of Hsp90.

\subsection{Statistical analyses}

The data are presented as mean values $\pm \mathrm{SD}$, and each value represents the mean of the results of at least three repetitive experiments in each group. A non-parametric test was performed using GraphPad Prism 7.0 to assess the significance of the difference between two groups, ${ }^{* * *} p<0.01$.

\section{Conclusion}

In summary, herein, we developed an easily synthesized BZ nanomaterial for use in nanomedicine delivery. The synthetic method improves the efficiency of the synthesis process, showing a broad application prospect. The synthesized nanomaterial had good light absorption potential and photothermal cycling stability in the near-infrared second region and exhibited profound biocompatibility. GA, a natural inhibitor of Hsp90, was then loaded onto the synthesized BZ nanomaterials with high efficiency. Owing to the GA-induced downmodulation of Hsp90 to overcome the thermoresistance of cancer cells, highly effective in vivo apoptosis of tumors was realized using GBZ in the low-temperature PTT at $43^{\circ} \mathrm{C}$.

\section{Conflicts of interest}

The authors declare no conflict of interest.

\section{Acknowledgements}

The manuscript was written through contributions of all authors. All authors have provided approval to the final version of the manuscript. This work was supported by the Hubei Province Health and Family Planning Scientific Research Project (WJ2017Z007), the Special Fund for Basic Scientific Research Operating Expenses of Central Universities (2042019kf0327), the National Research and Development Program for Major Research Instruments (Grant No. 81527801), the National Key Research and Development Program (2016YFC1000701), the Basic Research Projects of Shenzhen Knowledge Innovation Program (JCYJ20180302173424902), and the Frontier Projects of Applied
Foundation in Wuhan (2019010701011386). The authors thank Huang Fei from the Shiyanjia Lab (http://www.shiyanjia.com).

\section{References}

1 J. D. Yang, P. Hainaut, G. J. Gores, A. Amadou, A. Plymoth and L. R. Roberts, A global view of hepatocellular carcinoma: trends, risk, prevention and management, Nat. Rev. Gastroenterol. Hepatol., 2019, 16(10), 589-604.

2 A. T. Tan and S. Schreiber, Adoptive T-cell therapy for HBVassociated HCC and HBV infection, Antiviral Res., 2020, 176, 104748.

3 P. R. Galle, F. Tovoli, F. Foerster, M. A. Worns, A. Cucchetti and L. Bolondi, The treatment of intermediate stage tumours beyond TACE: From surgery to systemic therapy, J. Hepatol., 2017, 67(1), 173-183.

4 M. Kudo, Systemic Therapy for Hepatocellular Carcinoma: 2017 Update, Oncology, 2017, 93(Suppl 1), 135-146.

5 L. Cerrito, B. E. Annicchiarico, R. Iezzi, A. Gasbarrini, M. Pompili and F. R. Ponziani, Treatment of hepatocellular carcinoma in patients with portal vein tumor thrombosis: Beyond the known frontiers, World J. Gastroenterol., 2019, 25(31), 4360-4382.

6 P. H. Liu, T. I. Huo and R. A. Miksad, Hepatocellular Carcinoma with Portal Vein Tumor Involvement: Best Management Strategies, Semin. Liver Dis., 2018, 38(3), 242-251.

7 P. Huang, P. Rong, A. Jin, X. Yan, M. G. Zhang, J. Lin, et al., Dye-loaded ferritin nanocages for multimodal imaging and photothermal therapy, Adv. Mater., 2014, 26(37), 64016408.

8 Y. Liu, P. Bhattarai, Z. Dai and X. Chen, Photothermal therapy and photoacoustic imaging via nanotheranostics in fighting cancer, Chem. Soc. Rev., 2019, 48(7), 2053-2108.

9 D. Zhu, Y. Duo, S. Meng, Y. Zhao, L. Xia, Y. Li, et al., Tumor-Exocytosed Exosome/Aggregation-Induced Emission Luminogen Hybrid Nanovesicles Facilitate Efficient Tumor Penetration and Photodynamic Therapy, Angew. Chem., 2020, 59, 2-10.

10 K. Cai, W. Zhang, J. Zhang, H. Li, H. Han and T. Zhai, Design of Gold Hollow Nanorods with Controllable Aspect Ratio for Multimodal Imaging and Combined ChemoPhotothermal Therapy in the Second Near-Infrared Window, ACS Appl. Mater. Interfaces, 2018, 10(43), 3670336710.

11 W. Feng, X. Han, R. Wang, X. Gao, P. Hu, W. Yue, et al., Nanocatalysts-Augmented and Photothermal-Enhanced Tumor-Specific Sequential Nanocatalytic Therapy in Both NIR-I and NIR-II Biowindows, Adv. Mater., 2019, 31(5), e1805919.

12 H. Lin, S. Gao, C. Dai, Y. Chen and J. Shi, A TwoDimensional Biodegradable Niobium Carbide (MXene) for Photothermal Tumor Eradication in NIR-I and NIR-II Biowindows, J. Am. Chem. Soc., 2017, 139(45), 16235-16247.

13 C. Yang, D. Xu, W. Peng, Y. Li, G. Zhang, F. Zhang, et al., Correction: Ti2C3Tx nanosheets as photothermal agents 
for near-infrared responsive hydrogels, Nanoscale, 2018, 10(36), 17409.

14 G. Zhang, H. Gou, Y. Liu, K. Xi, D. Jiang and X. Jia, pHresponsive PEG-chitosan/iron oxide hybrid nanoassemblies for low-power-assisted PDT/PTT combination therapy, Nanomedicine, 2020, 15(11), 1097-1112.

15 L. C. Mimun, G. Ajithkumar, M. Pokhrel, B. G. Yust, Z. G. Elliott, F. Pedraza, et al., Bimodal Imaging Using Neodymium Doped Gadolinium Fluoride Nanocrystals with Near-Infrared to Near-Infrared Downconversion Luminescence and Magnetic Resonance Properties, J. Mater. Chem. B, 2013, 1(41), 5702-5710.

16 X. Wang, G. Ku, M. A. Wegiel, D. J. Bornhop, G. Stoica and L. V. Wang, Noninvasive photoacoustic angiography of animal brains in vivo with near-infrared light and an optical contrast agent, Opt. Lett., 2004, 29(7), 730-732.

17 Y. Cao, T. Wu, K. Zhang, X. Meng, W. Dai, D. Wang, et al., Engineered Exosome-Mediated Near-Infrared-II Region V2C Quantum Dot Delivery for Nucleus-Target Low-Temperature Photothermal Therapy, ACS Nano, 2019, 13(2), 1499-1510.

18 K. Welsher, Z. Liu, S. P. Sherlock, J. T. Robinson, Z. Chen, D. Daranciang, et al., A route to brightly fluorescent carbon nanotubes for near-infrared imaging in mice, Nat. Nanotechnol., 2009, 4(11), 773-780.

19 S. Zhu, R. Tian, A. L. Antaris, X. Chen and H. Dai, NearInfrared-II Molecular Dyes for Cancer Imaging and Surgery, Adv. Mater., 2019, 31(24), e1900321.

20 Y. Yang, W. Zhu, Z. Dong, Y. Chao, L. Xu, M. Chen, et al., 1D Coordination Polymer Nanofibers for Low-Temperature Photothermal Therapy, Adv. Mater., 2017, 29, 40.

21 S. Shen, L. Feng, S. Qi, J. Cao, Y. Ge, L. Wu, et al., Reversible Thermochromic Nanoparticles Composed of a Eutectic Mixture for Temperature-Controlled Photothermal Therapy, Nano Lett., 2020, 20(3), 2137-2143.

22 Z. Yuan, C. Lin, Y. He, B. Tao, M. Chen, J. Zhang, et al., Near-Infrared Light-Triggered Nitric-Oxide-Enhanced Photodynamic Therapy and Low-Temperature Photothermal Therapy for Biofilm Elimination, ACS Nano, 2020, 14(3), 3546-3562.

23 J. L. Bruce, B. D. Price, C. N. Coleman and S. K. Calderwood, Oxidative injury rapidly activates the heat shock transcription factor but fails to increase levels of heat shock proteins, Cancer Res., 1993, 53(1), 12-15.

24 C. Zhang, K. Zhao, W. Bu, D. Ni, Y. Liu, J. Feng, et al., Marriage of scintillator and semiconductor for synchronous radiotherapy and deep photodynamic therapy with diminished oxygen dependence, Angew. Chem., Int. Ed., 2015, 54(6), 1770-1774.

25 L. An, M. Cao, X. Zhang, J. Lin, Q. Tian and S. Yang, pH and Glutathione Synergistically Triggered Release and SelfAssembly of Au Nanospheres for Tumor Theranostics, ACS Appl. Mater. Interfaces, 2020, 12(7), 8050-8061.

26 Y. Cai, Y. Zhang, S. Ji, Y. Ye, S. Wu, J. Liu, et al., Laser ablation in liquids for the assembly of Se@Au chain-oligomers with long-term stability for photothermal inhibition of tumor cells, J. Colloid Interface Sci., 2020, 566, 284-295.
27 W. Kong, Q. Wang, G. Deng, H. Zhao, L. Zhao, J. Lu, et al., Se@SiO2@Au-PEG/DOX NCs as a multifunctional theranostic agent efficiently protect normal cells from oxidative damage during photothermal therapy, Dalton Trans., 2020, 49(7), 2209-2217.

28 L. Dong, G. Ji, Y. Liu, X. Xu, P. Lei, K. Du, et al., Multifunctional $\mathrm{Cu}-\mathrm{Ag} 2 \mathrm{~S}$ nanoparticles with high photothermal conversion efficiency for photoacoustic imagingguided photothermal therapy in vivo, Nanoscale, 2018, 10(2), 825-831.

29 M. Hao, C. Kong, C. Jiang, R. Hou, X. Zhao, J. Li, et al., Polydopamine-coated Au-Ag nanoparticle-guided photothermal colorectal cancer therapy through multiple cell death pathways, Acta Biomater., 2019, 83, 414-424.

30 X. Li, Z. Liu, K. Luo, X. Yin, X. Lin and C. Zhu, Biomimetic Synthesis of Ag2 Se Quantum Dots with Enhanced Photothermal Properties and as "Gatekeepers" to Cap Mesoporous Silica Nanoparticles for Chemo-Photothermal Therapy, Chem. - Asian J., 2019, 14(1), 155-161.

31 Y. Dou, X. Li, W. Yang, Y. Guo, M. Wu, Y. Liu, et al., PB@Au Core-Satellite Multifunctional Nanotheranostics for Magnetic Resonance and Computed Tomography Imaging in Vivo and Synergetic Photothermal and Radiosensitive Therapy, ACS Appl. Mater. Interfaces, 2017, 9(2), 1263-1272.

32 H. Li, W. Zhang, L. Ding, X. W. Li, Y. Wu and J. H. Tang, Prussian blue-modified ferritin nanoparticles for effective tumor chemo-photothermal combination therapy via enhancing reactive oxygen species production, J. Biomater. Appl., 2019, 33(9), 1202-1213.

33 Y. Y. Su, Z. Teng, H. Yao, S. J. Wang, Y. Tian, Y. L. Zhang, et al., A Multifunctional PB@mSiO2-PEG/DOX Nanoplatform for Combined Photothermal-Chemotherapy of Tumor, ACS Appl. Mater. Interfaces, 2016, 8(27), 1703817046.

34 P. Lei, R. An, X. Zheng, P. Zhang, K. Du, M. Zhang, et al., Ultrafast synthesis of ultrasmall polyethylenimine-protected AgBiS2 nanodots by "rookie method" for in vivo dual-modal CT/PA imaging and simultaneous photothermal therapy, Nanoscale, 2018, 10(35), 16765-16774.

35 L. Song, X. Dong, S. Zhu, C. Zhang, W. Yin, X. Zhang, et al., Bi2 S3 -Tween 20 Nanodots Loading PI3 K Inhibitor, LY294002, for Mild Photothermal Therapy of LoVo Cells In Vitro and In Vivo, Adv. Healthcare Mater., 2018, 7(22), e1800830.

36 S. Wang, H. Wang, C. Song, Z. Li, Z. Wang, H. Xu, et al., Synthesis of Bi2WO6-x nanodots with oxygen vacancies as an all-in-one nanoagent for simultaneous CT/IR imaging and photothermal/photodynamic therapy of tumors, Nanoscale, 2019, 11(32), 15326-15338.

37 J. Liu, P. Wang, X. Zhang, L. Wang, D. Wang, Z. Gu, et al., Rapid Degradation and High Renal Clearance of Cu3BiS3 Nanodots for Efficient Cancer Diagnosis and Photothermal Therapy in Vivo, ACS Nano, 2016, 10(4), 4587-4598.

38 J. Bai, X. Jia, Y. Ruan, C. Wang and X. Jiang, Photosensitizer-Conjugated Bi2Te3 Nanosheets as Theranostic Agent for Synergistic Photothermal and 
Photodynamic Therapy, Inorg. Chem., 2018, 57(16), 1018010188.

39 F. Mao, L. Wen, C. Sun, S. Zhang, G. Wang, J. Zeng, et al., Ultrasmall Biocompatible Bi2Se3 Nanodots for Multimodal Imaging-Guided Synergistic Radiophotothermal Therapy against Cancer, ACS Nano, 2016, 10(12), 11145-11155.

40 S. Yang, Z. Li, Y. Wang, X. Fan, Z. Miao, Y. Hu, et al., Multifunctional Bi@PPy-PEG Core-Shell Nanohybrids for Dual-Modal Imaging and Photothermal Therapy, ACS Appl. Mater. Interfaces, 2018, 10(2), 1605-1615.

41 X. Yu, A. Li, C. Zhao, K. Yang, X. Chen and W. Li, Ultrasmall Semimetal Nanoparticles of Bismuth for DualModal Computed Tomography/Photoacoustic Imaging and Synergistic Thermoradiotherapy, ACS Nano, 2017, 11(4), 3990-4001.

42 C. Yan, H. Zhao, J. Li, H. Jin, L. Liu, W. Wu, et al., MildTemperature Solution-Assisted Encapsulation of Phosphorus into ZIF-8 Derived Porous Carbon as LithiumIon Battery Anode, Small, 2020, 16(11), e1907141.

43 F. Zou, J. Jiang, F. Lv, X. Xia and X. Ma, Preparation of antibacterial and osteoconductive 3D-printed PLGA/Cu(I) (aZIF-8 nanocomposite scaffolds for infected bone repair, J. Nanobiotechnol., 2020, 18(1), 39.

44 G. Luo, S. Jiang, X. Zhang, Y. Ling, H. Luo and Y. Zhang, Gambogic Acid Affects Ribosomal Occurrence in Glioma Cells by Downregulating the Phosphoinositide Kinase-3/ Protein Kinase B/Mammalian Target of Rapamycin Signaling Pathway, J. Nanosci. Nanotechnol., 2020, 20(6), 3361-3372.

45 T. Costa, N. M. Raghavendra and C. Penido, Natural heat shock protein 90 inhibitors in cancer and inflammation, Eur. J. Med. Chem., 2020, 189, 112063.
46 Y. Wang, X. Ji, P. Pang, Y. Shi, J. Dai, J. Xu, et al., Synthesis of Janus Au nanorods/polydivinylbenzene hybrid nanoparticles for chemo-photothermal therapy, J. Mater. Chem. $B, 2018,6(16), 2481-2488$.

47 C. M. Hessel, V. P. Pattani, M. Rasch, M. G. Panthani, B. Koo, J. W. Tunnell, et al., Copper selenide nanocrystals for photothermal therapy, Nano Lett., 2011, 11(6), 25602566.

48 E. B. Kang, J. E. Lee, Z. A. I. Mazrad, I. In, J. H. Jeong and S. Y. Park, pH-Responsible fluorescent carbon nanoparticles for tumor selective theranostics via $\mathrm{pH}$-turn on/off fluorescence and photothermal effect in vivo and in vitro, Nanoscale, 2018, 10(5), 2512-2523.

49 X. Li, J. Zhou, X. Dong, W. Y. Cheng, H. Duan and P. C. K. Cheung, In Vitro and In Vivo Photothermal Cancer Therapeutic Effects of Gold Nanorods Modified with Mushroom beta-Glucan, J. Agric. Food Chem., 2018, 66(16), 4091-4098.

50 A. Mohammadi Gazestani, S. Khoei, S. Khoee, S. Emamgholizadeh Minaei and M. Motevalian, In vivo evaluation of the combination effect of near-infrared laser and 5-fluorouracil-loaded PLGA-coated magnetite nanographene oxide, Artif. Cells, Nanomed., Biotechnol., 2018, 46(sup2), 25-33.

51 J. Liu, X. Zheng, L. Yan, L. Zhou, G. Tian, W. Yin, et al., Bismuth sulfide nanorods as a precision nanomedicine for in vivo multimodal imaging-guided photothermal therapy of tumor, ACS Nano, 2015, 9(1), 696-707.

52 D. M. Zhu, W. Xie, Y. S. Xiao, M. Suo, M. H. Zan, Q. Q. Liao, et al., Erythrocyte membrane-coated gold nanocages for targeted photothermal and chemical cancer therapy, Nanotechnology, 2018, 29(8), 084002. 